\title{
THE CONVERGENCE PART OF A KNINTCHINE-TYPE THEOREM IN THE RING OF ADELES
}

\author{
Ella Kovalevskaya
}

\begin{abstract}
We prove the convergence part of a Khintchine-type theorem for simultaneous Diophantine approximation of zero by values of integral polynomials at the points

$$
\left(x, z, \omega_{1}, \omega_{2}\right) \in \mathbb{R} \times \mathbb{C} \times \mathbb{Q}_{p_{1}} \times \mathbb{Q}_{p_{2}},
$$

where $p_{1} \neq p_{2}$ are primes. It is a generalization of Sprindžuk's problem (1980) in the ring of adeles. We continue our investigation (2013), where the problem was proved at the points in $\mathbb{R}^{2} \times \mathbb{C} \times \mathbb{Q}_{p_{1}}$. We use the most precise form of the essential and inessential domains method in metric theory of Diophantine approximation.
\end{abstract}

\section{Introduction}

We investigate the convergence part of a Khintchine-type theorem for simultaneous Diophantine approximation of zero by values of integral polynomials $P, \operatorname{deg} P=n$, at the points

$$
\left(x, z, \omega_{1}, \omega_{2}\right) \in \mathbb{R} \times \mathbb{C} \times \mathbb{Q}_{p_{1}} \times \mathbb{Q}_{p_{2}},
$$

where $p_{1} \neq p_{2}$ are primes, and $n \geq 4$. According to contemporary therminology it is Diophantine approximation in the ring of adeles. The problem can be viewed as a generalization of S prind žuk's problem (1980). It arises from studies of real numbers that are badly- or well-approximable by rational numbers.

Let $P=P(t)=a_{n} t^{n}+\cdots+a_{1} t+a_{0} \in \mathbb{Z}[t], a_{n} \neq 0, H=H(P)=$ $\max \left(\left|a_{n}\right|, \ldots,\left|a_{0}\right|\right)$. Let $p_{i} \geq 2, \mathbb{Q}_{p_{i}}$ be the field of $p_{i}$-adic numbers, $|\cdot|_{p_{i}}$ be the $p_{i}$-adic valuation $(i=1,2)$. Suppose that $\mathcal{O}=\mathbb{R} \times \mathbb{C} \times \mathbb{Q}_{p_{1}} \times \mathbb{Q}_{p_{2}}$. We define a measure $\bar{\mu}$ in $\mathcal{O}$ as a product of the Lebesque measure $\mu_{1}$ in $\mathbb{R}$, the Lebesque measure $\mu_{2}$ in $\mathbb{C}$ and the Haar measures $\mu_{p_{i}}$ in $\mathbb{Q}_{p_{i}}(i=1,2)$,

(C) 2014 Mathematical Institute, Slovak Academy of Sciences.

2010 Mathematics Subject Classification: 11J61, 11J83, 11K60.

Keywords: metric theory of Diophantine approximation, ring of adeles, roots of integral polynomials. 


\section{ELLA KOVALEVSKAYA}

i.e., $\bar{\mu}=\mu_{1} \mu_{2} \mu_{p_{1}} \mu_{p_{2}}$. Let $\Psi: \mathbb{N} \rightarrow \mathbb{R}^{+}, \Psi \in \mathcal{C}(\mathbb{R})$ be a monotonic decreasing function, $\Lambda=\left(\lambda_{1}, \lambda_{2}, \lambda_{3}, \lambda_{4}\right), V=\left(v_{1}, v_{2}, v_{3}, v_{4}\right)$ are vectors in $\mathbb{R}^{4}$, and $\lambda_{i} \geq 0$, $v_{i} \geq 0$. Consider the system of inequalities

$$
\begin{array}{rlrl}
|P(x)| & <H^{-v_{1}} \Psi(H)^{\lambda_{1}}, & |P(z)|<H^{-v_{2}} \Psi(H)^{\lambda_{2}}, \\
\left|P\left(\omega_{1}\right)\right|_{p_{1}}<H^{-v_{3}} \Psi(H)^{\lambda_{3}}, & \left|P\left(\omega_{2}\right)\right|_{p_{2}}<H^{-v_{4}} \Psi(H)^{\lambda_{4},}
\end{array}
$$

where $\left(x, z, \omega_{1}, \omega_{2}\right) \in \mathcal{O}$ and $v_{1}+2 v_{2}+v_{3}+v_{4}=n-4, \lambda_{1}+2 \lambda_{2}+\lambda_{3}+\lambda_{4}=1$. Let $M_{n}(V, \Psi, \Lambda)$ be a set of the points $\left(x, z, \omega_{1}, \omega_{2}\right) \in \mathcal{O}$ for which the system (1) has infinitely many solutions in polynomials $P \in \mathbb{Z}[t], \operatorname{deg} P=n$. We prove

Theorem. If $n \geq 4$ and $\sum_{H=1}^{\infty} \Psi(H)<\infty$, then $\bar{\mu}\left(M_{n}(V, \Psi, \Lambda)\right)=0$.

Another words, the theorem asserts that the system (11) has only a finite set of solutions in $P \in \mathbb{Z}[t], \operatorname{deg} P=n$, for almost all points in $\mathcal{O}$ under the formulated condition on the parameters and the function $\Psi$.

The proof of the theorem is obtained by applying the most precise form of the essential and inessential domains method introduced by S p r ind ž u k (1964). This method is being developed to this day, with most contributions coming from the number theory schools at the National Academy of Sciences of Belarus (Minsk, Belarus) and the University of York (York, UK) [1]- [] , [], [9].

Here we continue our investigation [6], where the problem was proved in $\mathbb{R}^{2} \times \mathbb{C} \times \mathbb{Q}_{p_{1}}$. We remark that in [10] the convergence part of an $S$-arithmetic $\mathbb{Z}_{s}$-Khintchine-type theorem for product of non-degenerate analytic manifolds in $\prod_{j=1}^{s} \mathbb{Q}_{p_{j}}$ was proved by applying the dynamic version $\mathrm{K}$ le i n b o c k-M a rg u li s lemma (1988).

The divergence part of a Khintchine-type theorem in $\mathbb{R} \times \mathbb{C} \times \mathbb{Q}_{p}$ was proved by N. B u d ar in a and E. Z or in (2009). The divergence part of our theorem will be proved in the next paper.

\section{Sketch of proof}

Our investigation is based on the method [7], the argumentations from [1] [ 6], [8], 9] and their development. Here we mark the main moments of proving and indicate the distinctions from [6].

Let $\mathbf{T}=I \times K \times D_{p_{1}} \times D_{p_{2}} \subset \mathcal{O}$, where $I$ is an interval in $\mathbb{R}, K$ is a circle in $\mathbb{C}$ and $D_{p_{i}}$ is a disc in $\mathbb{Q}_{p_{i}}(i=1,2)$, be an elementary set in $\mathcal{O}$. We call it as a parallelepiped. According to a metric character of the theorem we will prove it for the points of $\mathbf{T}, \bar{\mu}(\mathbf{T})=1$. Fix $\delta>0$ and exclude from $\mathbf{T}$ a set of the points $\left(x, z, \omega_{1}, \omega_{2}\right)$ which satisfy the inequalities: $|x|<\delta,|\operatorname{Im} z|<\delta$ and $|\omega|_{p_{i}}<\delta$ $(i=1,2)$. Thus, from now on we will assume that the points $\left(x, z, \omega_{1}, \omega_{2}\right) \in \mathbf{T}$ satisfy the condition: $|x| \geq \delta,|\operatorname{Im} z| \geq \delta$ and $|\omega|_{p_{i}} \geq \delta(i=1,2)$. Without loss of generality we assume that $\delta$ is arbitrary small. 


\section{THE CONVERGENCE PART OF A KNINTCHINE-TYPE THEOREM}

Introduce a class of polynomials

$$
\mathcal{P}_{n}(Q)=\{P \in \mathbb{Z}[t]: H(P) \leq Q\}, \quad \text { where } \quad Q>Q_{0}>0 .
$$

The important moment of the proof is a reduction to irreducible and leading polynomials $P \in \mathcal{P}_{n}(Q)$. Denote a set of such polynomials $P$ as $\mathfrak{P}_{n}$.

A polynomial $P$ with the leading coefficient $a_{n}$ will be called leading if $\left|a_{n}\right| \leq H(P)<c(n)\left|a_{n}\right|$, where the constant $c(n) \geq 1$ depends only of $n$, and $\left.\left|a_{n}\right|_{p_{i}}>p_{i}^{-n}\right)$ (as [7, Ch. $1, \S 5, \S 6$ and Ch. 2, $\left.\S 2\right]$ or [1]).

Let $\mathfrak{P}_{n}(H)$ denote a set of polynomials $P \in \mathfrak{P}_{n}$ satisfying (1) for which $H(P)=H$, where $H$ is a fix number, $0<Q_{0}<H \leq Q$ and $Q_{0}$ is sufficiently large. Then the set $\mathfrak{P}_{n}(H)$ is divided into $\varepsilon$-classes $\mathfrak{P}_{n}\left(H, \mathbf{q}, \mathbf{r}, \mathbf{s}_{1}, \mathbf{s}_{2},\right)$ according to the distances between their roots $(\S 3$, formulas $(2),(3)$ and the text above and below these formulas). Next, we prove the theorem for each $\varepsilon$-class. For this, we introduce the notion of $\left(i_{1}, i_{2}, i_{3}, i_{4}\right)$-linear polynomial, where $i_{j} \in\{0,1\}$ $(j=1,2,3,4)$. For example, (0,0,0,0)-linear polynomial, $(1,1,1,1)$-linear one, $(0,1,1,0)$-linear one and so on). We have 16 cases of linearity. This notion is necessary to obtain the lower bounds for the derivatives $\left|P^{\prime}(x)\right|,\left|P^{\prime}(z)\right|$ and $\left|P^{\prime}\left(\omega_{i}\right)\right|_{p_{i}}(i=1,2)$ of $P \in \mathfrak{P}_{n}(H)$. On the other hand, Lemma $2 \S 3$ gives the upper bounds for them. Fix an admissible vector $\left(i_{1}, i_{2}, i_{3}, i_{4}\right)$. Let $\mathfrak{P}_{n}^{\left(i_{1}, i_{2}, i_{3}, i_{4}\right)}$ be a class of $\left(i_{1}, i_{2}, i_{3}, i_{4}\right)$-linear polynomials $P$ in $\mathfrak{P}_{n}\left(H, \mathbf{q}, \mathbf{r}, \mathbf{s}_{1}, \mathbf{s}_{2}\right)$.

Now, we fix $P \in \mathfrak{P}_{n}^{\left(i_{1}, i_{2}, i_{3}, i_{4}\right)}$ and construct a countable covering of $M_{n}(V, \Psi, \Lambda)$ by the system of the small parallelepipeds $\Pi_{j}(P) \subset \mathbf{T}(j=1,2, \ldots)$, i.e., $M_{n}(V, \Psi, \Lambda) \subseteq \sum_{j} \Pi_{j}(P)$. These parallelepipeds $\Pi_{j}(P)$ are divided into two classes: the essential and the inessential (analogously to [7, $\S 10, \S 11]$ ).

Definition 1. The parallelepiped $\Pi_{j}(P)$ is called essential if for all polynomials

$$
P_{j} \neq P, \quad P_{j} \in \mathfrak{P}_{n}^{\left(i_{1}, i_{2}, i_{3}, i_{4}\right)},
$$

we have

If there exists

$$
\bar{\mu}\left(\Pi_{j}(P) \bigcap \Pi_{j}\left(P_{j}\right)\right)<\frac{1}{2} \mu \Pi_{j}(P) .
$$

$$
P_{j} \in \mathfrak{P}_{n}^{\left(i_{1}, i_{2}, i_{3}, i_{4}\right)}, \quad P_{j} \neq P,
$$

such that

$$
\bar{\mu}\left(\Pi_{j}(P) \bigcap \Pi_{j}\left(P_{j}\right)\right) \geq \frac{1}{2} \mu \Pi_{j}(P),
$$

then the parallelepiped $\Pi_{j}(P)$ is called inessential.

Next, as well as in [1]- 9] using Lemmas 1-4 $\S 3$ and the classic metric Borel-Cantelli theorem [7, Ch. 1, $\S 3$, Lemma 12] we show that the measure of the set of points lying in infinitely many essential parallelepipeds $\Pi_{j}(P)$ equals zero, and the same is true for measure of the set of points lying in infinitely many inessential parallelepipeds $\Pi_{j}(P)$. 


\section{ELLA KOVALEVSKAYA}

\section{Lemmas on polynomials}

Fix $P \in \mathfrak{P}_{n}(H)$. Let $P$ has roots $\alpha_{1}, \alpha_{1}, \ldots, \alpha_{n}$ in $\mathbb{C}$ and roots $\gamma_{i 1}, \gamma_{i 2}, \ldots, \gamma_{i n}$ in $\mathbb{Q}_{p_{i}}^{*}, i=1,2$, where $\mathbb{Q}_{p_{i}}^{*}$ is the smallest field containing $\mathbb{Q}_{p_{i}}$ and all algebraic numbers. As usual, $X \ll Y$ is equivalent to $X=O(Y)$. According to Lemma 1 [7. Ch. 1, $\S 2]$ and Lemma 4 [7, Ch. 2, $\S 2]$ we have

$$
\left|\alpha_{j}\right| \ll 1, \quad\left|\gamma_{i 1}\right|_{p_{1}} \ll 1 \text { and }\left|\gamma_{i 2}\right|_{p_{2}} \ll 1, \quad j=1, \ldots, n \text {. }
$$

Let $\alpha_{1}, \ldots, \alpha_{k}$ be real roots of $P$ and $\beta_{1}, \ldots, \beta_{(n-k) / 2}$ be its complex roots. Since $P$ is irreducible, then all of its roots are different. Let $\left(\alpha_{i 1}, \alpha_{i 2}, \ldots, \alpha_{i n}\right)$ be a permutation of $\left(\alpha_{1}, \alpha_{1}, \ldots, \alpha_{n}\right)$. Choose real root $\alpha_{i 1}=\alpha_{1} \in I$, a complex root $\beta_{1}=\alpha_{i 2} \in K$, and two $p_{i}$-adic roots $\gamma_{i 1} \in D_{p_{1}}, \gamma_{i 2} \in D_{p_{2}}$. Remember that the definition of the parallelepiped $\mathbf{T}=I \times K \times D_{p_{1}} \times D_{p_{2}}$ was introduced at the beginning of $\S 2$. Define the sets

$$
S_{i}\left(\alpha_{j i}\right)=\left\{u \in \mathbb{U}:\left|u-\alpha_{j i}\right|=\min _{1 \leq k \leq n}\left|u-\alpha_{k}\right|\right\}, \quad i=1,2,
$$

where $u$ represents $x$ or $z$, and $\alpha_{j i}$ is real or complex root of $P$, and $\mathbb{U}$ is $I \subset \mathbb{R}$ or $K \subset \mathbb{C}$ as required, and

$$
S_{p_{i}}\left(\gamma_{j i}\right)=\left\{\omega_{i} \in D_{p_{i}} \subset \mathbb{Q}_{p_{i}}:\left|\omega_{i}-\gamma_{i j}\right|_{p}=\min _{1 \leq k \leq n}\left|\omega_{i}-\gamma_{i k}\right|_{p_{i}}\right\}, \quad i=1,2 .
$$

Consider these sets for a fixed vector $\left(\alpha_{j 1}, \alpha_{j 2}, \gamma_{j 1}, \gamma_{j 2}\right)$ and for simplicity assume that $\alpha_{j 1}=\alpha_{1}, \alpha_{j 2}=\beta_{1}, \gamma_{j 1}=\gamma_{1}$ and $\gamma_{j 2}=\gamma_{2}$. Reorder the other roots of $P$ in the following way:

(1) $\left|\alpha_{1}-\alpha_{2}\right| \leq\left|\alpha_{1}-\alpha_{3}\right| \leq \cdots \leq\left|\alpha_{1}-\alpha_{k}\right|$,

(2) $\left|\beta_{1}-\beta_{2}\right| \leq \cdots \leq\left|\beta_{1}-\beta_{(n-k) / 2}\right|$,

(3) $\left|\gamma_{1}-\gamma_{12}\right|_{p_{1}} \leq \cdots \leq\left|\gamma_{1}-\gamma_{1 n}\right|_{p_{1}}$,

(4) $\left|\gamma_{2}-\gamma_{22}\right|_{p_{2}} \leq \cdots \leq\left|\gamma_{2}-\gamma_{2 n}\right|_{p_{2}}$.

Also, for the given polynomial $P \in \mathfrak{P}_{n}(H)$ we define numbers $\rho_{i j} \in \mathbb{R}$ by $\left|\alpha_{i 1}-\alpha_{i j}\right|=H^{-\rho_{i j}}, 2 \leq j \leq n, \rho_{i n} \leq \rho_{i 2} \leq \cdots \leq \rho_{i 2}(i=1,2,3,4)$, where $\alpha_{11}=\alpha_{1}, \alpha_{21}=\beta_{1}, \alpha_{31}=\gamma_{1}$ and $\alpha_{41}=\gamma_{2}$. Since all of the roots are bounded (see the beginning $\S 3$ ), then there exists $\varepsilon_{1}>1$ such that $\rho_{i j} \geq-\varepsilon_{1} / 2$ for $i=1,2,3,4$ and $j=2,3, \ldots, n$. Choose $\varepsilon>0$ such that $\varepsilon_{1}=\varepsilon / T_{1}$ for some sufficiently large $T_{1}>T_{0}>0$. Let $T=\left[n / \varepsilon_{1}\right]+1$. Define the integers $\left(k_{1}, l_{j}, m_{1 j}, m_{2 j}\right)=\left(t_{1 j}, t_{2 j}, t_{3 j}, t_{4 j}\right)(j=2,3, \ldots, n)$ by the relations

$$
\left(t_{i j}-1\right) / T \leq \rho_{i j}<t_{i j} / T, \quad t_{i 2} \geq t_{i 3} \geq \cdots \geq t_{i n} \geq 0, \quad i=1,2,3,4 .
$$




\section{THE CONVERGENCE PART OF A KNINTCHINE-TYPE THEOREM}

Finally, define the numbers $q_{i}, r_{i}$ and $s_{1 i}, s_{2 i}(i=1,2, \ldots, n-1)$ by the formulas

$$
q_{i}=T^{-1} \sum_{t=i+1}^{n} k_{t}, \quad r_{i}=T^{-1} \sum_{t=i+1}^{n} l_{t}, \quad s_{1 i}=T^{-1} \sum_{t=i+1}^{n} m_{1 t}, \quad s_{2 i}=T^{-1} \sum_{t=i+1}^{n} m_{2 t} .
$$

Each polynomial $P \in \mathfrak{P}_{n}(H)$ is now associated with four vectors:

$$
\begin{gathered}
\mathbf{q}=\left(q_{1}, q_{2}, \ldots, q_{n-1}\right), \\
\mathbf{r}=\left(r_{1}, r_{2}, \ldots, r_{n-1}\right), \\
\mathbf{s}_{1}=\left(s_{11}, s_{12}, \ldots, s_{1, n-1}\right), \\
\mathbf{s}_{2}=\left(s_{21}, s_{22}, \ldots, s_{2, n-1}\right) .
\end{gathered}
$$

The number of these vectors is finite and depends only on $n, \rho$ and $T$ (see [7, Ch. 1, Lemma 24 and Ch. 2, Lemma 12]). Let $\mathfrak{P}_{n}\left(H, \mathbf{q}, \mathbf{r}, \mathbf{s}_{1}, \mathbf{s}_{2}\right)$ denote the set of polynomials $P \in \mathfrak{P}_{n}(H)$ having the same four vectors $\left(\mathbf{q}, \mathbf{r}, \mathbf{s}_{1}, \mathbf{s}_{2}\right)$. Thus, we divide the set $\mathfrak{P}_{n}(H)$ into $\varepsilon$-classes $\mathfrak{P}_{n}\left(H, \mathbf{q}, \mathbf{r}, \mathbf{s}_{1}, \mathbf{s}_{2}\right)$.

Now without loss of generality we assumed that $x \in S_{1}\left(\alpha_{1}\right) \subset I, z \in S_{2}\left(\beta_{1}\right) \subset K$, $\omega_{1} \in S_{p_{1}}\left(\gamma_{11}\right) \subset D_{p_{1}}$ and $\omega_{2} \in S_{p_{2}}\left(\gamma_{21}\right) \subset D_{p_{2}}$. At many moments of our proof the values of the polynomials $P \in \mathfrak{P}_{n}\left(H, \mathbf{q}, \mathbf{r}, \mathbf{s}_{1}, \mathbf{s}_{2}\right)$ will be estimated by means of a Taylor series. To obtain an upper bounds of the terms in the Taylor series and for the other purposes the following two lemmas will be used.

LEMma 1. If $P \in \mathfrak{P}_{n}(H)$, then according to the notations of $\S 3$ we have

$$
\begin{aligned}
& |\widetilde{u}-\widetilde{\alpha}| \leq 2^{n}\left|P_{n}(\widetilde{u})\right|\left|P_{n}^{\prime}(\widetilde{\alpha})\right|^{-1}, \quad\left|\omega_{i}-\gamma_{i 1}\right|_{p_{i}} \leq\left|P_{n}\left(\omega_{i}\right)\right|_{p_{i}}\left|P_{n}^{\prime}\left(\gamma_{i 1}\right)\right|_{p_{i}}^{-1}, \quad i=1,2, \\
& \left\{\begin{array}{l}
|\widetilde{u}-\widetilde{\alpha}| \leq \min _{2 \leq j \leq n}\left(2^{n-j}\left(\left|P_{n}(\widetilde{u})\right|\left|P_{n}^{\prime}(\widetilde{\alpha})\right|^{-1} \prod_{k=2}^{j}\left|\widetilde{\alpha}-\alpha_{k}\right|\right)^{1 / j}\right), \\
\left|\omega_{i}-\gamma_{i 1}\right|_{p_{i}} \leq \min _{2 \leq j \leq n}\left(\left|P_{n}\left(\omega_{i}\right)\right|_{p_{i}}\left|P_{n}^{\prime}\left(\gamma_{i 1}\right)\right|_{p}^{-1} \prod_{k=2}^{j}\left|\gamma_{i 1}-\gamma_{i k}\right|_{p_{i}}\right)^{1 / j}, \quad i=1,2,
\end{array}\right.
\end{aligned}
$$

where $\widetilde{u}$ represents $x$ or $z$ and $\widetilde{\alpha}$ is $\alpha_{1}$ or $\beta_{1}$ as required.

Lemma 1 is proved in [1, 2], 9, pp. 36, 131].

Lemma 2. Let $P \in \mathfrak{P}_{n}\left(H, \boldsymbol{q}, \boldsymbol{r}, \boldsymbol{s}_{1}, \boldsymbol{s}_{2}\right)$. Then

$$
\begin{aligned}
& \left|P^{(l)}\left(\alpha_{1}\right)\right|<c(n) H^{1-q_{l}+(n-l) \varepsilon_{1}}, \\
& \left|P^{(l)}\left(\beta_{1}\right)\right|<c(n) H^{1-r_{l}+(n-l) \varepsilon_{1}}, \\
& \left|P^{(l)}\left(\gamma_{i 1}\right)\right|_{p_{i}}<c(n) H^{-s_{i l}+(n-l) \varepsilon_{1}}, \quad i=1,2, \quad 1 \leq l \leq n-1,
\end{aligned}
$$

where the constant $c(n)>0$ depends only on $n$.

The first and the second inequalities of lemma are proved in [1, [9, pp. 36-37]. The third and the fourth inequalities of it is proved in [4, Lemma 5]. 


\section{ELLA KOVALEVSKAYA}

There are various cases for $P \in \mathfrak{P}_{n}\left(H, \mathbf{q}, \mathbf{r}, \mathbf{s}_{1}, \mathbf{s}_{2}\right)$ to consider. Sometimes the existence of one case is disproved by finding a contradiction to the final inequality in the following lemma.

LEMma 3. Let $P_{1}, P_{2} \in \mathbb{Z}[t]$ be polynomials of degree at most $n$ with no common roots and $\max \left(H\left(P_{1}\right), H\left(P_{2}\right)\right) \leq H\left(H>Q_{0}\right)$. Let $\delta>0$ and $\eta_{j}>0, j=1,2,3,4$. Let $I \subset \mathbb{R}$ be an interval, $\mu_{1} I=H^{-\eta_{1}}, K \subset \mathbb{C}$ be circle, diam $K=H^{-\eta_{2}}$ and $D_{p_{i}} \subset \mathbb{Q}_{p_{i}}$ be a disk, $\mu_{p_{i}}\left(D_{p_{i}}\right)=H^{-\eta_{i+2}}, i=1,2$. If there exist $\tau_{i}>-1$ and $\tau_{i+2}>0, i=1,2$ such that for all $\left(x, z, \omega_{1}, \omega_{2}\right) \in I \times K \times D_{p_{1}}, \times D_{p_{2}}$ we have

$$
\begin{aligned}
\max \left(\left|P_{1}(x)\right|,\left|P_{2}(x)\right|\right) & <H^{-\tau_{1}}, \\
\max \left(\left|P_{1}(z)\right|,\left|P_{2}(z)\right|\right) & <H^{-\tau_{2}}, \\
\max \left(\left|P_{1}\left(\omega_{i}\right)\right|_{p_{i}},\left|P_{2}\left(\omega_{i}\right)\right|_{p_{i}}\right) & <H^{-\tau_{i+2}, \quad i=1,2,}
\end{aligned}
$$

then

$$
\begin{aligned}
\tau_{1}+2 \tau_{2}+\tau_{3}+\tau_{4}+3 & +2 \max \left(\tau_{1}+1-\eta_{1}, 0\right) \\
& +4 \max \left(\tau_{2}+1-\eta_{2}, 0\right) \\
& +2 \max \left(\tau_{3}+1-\eta_{3}, 0\right) \\
& +2 \max \left(\tau_{4}+1-\eta_{4}, 0\right)<2 n+\delta .
\end{aligned}
$$

$\mathrm{P}$ r o o f of the lemma is analogous to [3. Distinctions consist only in the sets of $\bar{X}=\left(X_{1}, X_{2}, X_{3}, X_{4}\right)$ and in the metrics of the corresponding spaces. Namely, in [3] we have $\bar{X}=(x, z, \omega) \in \mathbb{R} \times \mathbb{C} \times \mathbb{Q}_{p}$, in our case we have $\bar{X}=\left(x, z, \omega_{1}, \omega_{2}\right) \in \mathbb{R} \times \mathbb{C} \times \mathbb{Q}_{p_{1}} \times \mathbb{Q}_{p_{2}}$.

Briefly, the lemma shows that if the values of two polynomials are small at a given $I \times K \times D_{p_{1}} \times D_{p_{2}}$, then the parameters $\tau_{1}, \ldots, \tau_{4}$ and $\eta_{1}, \ldots, \eta_{4}$ are connected by the final inequality of lemma.

Lemma 4. Let $P \in \mathbb{Z}[t], \operatorname{deg} P=n \geq 4$ and $v>0$. Let $G(v)$ be the set of points $\left(x, z, \omega_{1}, \omega_{2}\right) \in \mathbb{R} \times \mathbb{C} \times \mathbb{Q}_{p_{1}} \times \mathbb{Q}_{p_{2}}$ for which the inequality

$$
|P(x)| \cdot|P(z)| \cdot\left|P\left(\omega_{1}\right)\right|_{p_{1}} \cdot\left|P\left(\omega_{2}\right)\right|_{p_{2}}<H^{-v}, \quad H=H(P),
$$

has infinitely many solutions $P$. Then $\bar{\mu} G(v)=0$ for $v>n-3$.

Lemma 4 is proved in [8].

Lemma 5. Let $H$ be a positive integer. Let us define a set of polynomials with integer coefficients $\mathcal{R}_{4}\left(b_{4}\right)=\left\{R(t) \in \mathbb{Z}[t], R(t)=b_{4} t^{4}+b_{3} t^{3}+b_{2} t^{2}+b_{1} t+b_{0}\right.$, where $\left.b_{4} \neq 0,\left|b_{i}\right| \leq\left|b_{4}\right| \leq H, i=0,1,2,3\right\}$. Let $\zeta_{j}>0, \varepsilon_{j}>0, j=1,2$. Take a nonempty open interval $I \subset \mathbb{R}$ and a nonempty complex ball $D \subset \mathbb{C}$ such that $I \cap D=\varnothing$. Then the system of inequalities

$$
|R(x)|<\zeta_{1}\left|b_{4}\right|^{-\varepsilon_{1}}, \quad x \in I ; \quad|R(z)|<\zeta_{2}\left|b_{4}\right|^{-\varepsilon_{2}}, \quad z \in D,
$$

holds for at most $c H$ polynomials in $\mathcal{R}_{4}\left(b_{4}\right)$, where the constant $c>0$ depends on $\zeta_{1}, \zeta_{2}$, the length of $I$ and the area of $D$. 


\section{THE CONVERGENCE PART OF A KNINTCHINE-TYPE THEOREM}

P r o of. The conditions of the lemma imply that there exist $\widetilde{x} \in I$ and $\widetilde{z} \in D$ such that (41) is satisfied and $\operatorname{Im} \widetilde{z}>\varepsilon$ for some $\varepsilon>0$, allowing us to write

$$
|\widetilde{x}-\widetilde{z}|>\varepsilon \text {. }
$$

Let $B\left(t_{0}, r\right)$ denote a ball in the complex plane with a center at $t_{0}$ of radius $r$. Let the coefficient $b_{3}$ be fixed. Then

$$
\begin{aligned}
& b_{2} \widetilde{x}^{2}+b_{1} \widetilde{x}+b_{0} \in B\left(x_{0}, \zeta_{1}\right), \\
& b_{2} \widetilde{z}^{2}+b_{1} \widetilde{z}+b_{0} \in B\left(z_{0}, \zeta_{2}\right),
\end{aligned}
$$

where

$$
x_{0}=-b_{4} \widetilde{x}^{4}-b_{3} \widetilde{x}^{3}, \quad z_{0}=-b_{4} \widetilde{z}^{4}-b_{3} \widetilde{z}^{3} .
$$

Subtracting the left-hand sides of the expressions (5) and (6), we have

$$
b_{2}(\widetilde{x}-\widetilde{z})(\widetilde{x}+\widetilde{z})+b_{1}(\widetilde{x}-\widetilde{z}) \in B\left(\left(x_{0}-z_{0}\right), \zeta_{1}+\zeta_{2}\right) .
$$

Dividing by $(\widetilde{x}-\widetilde{z})$ leads to

$$
\left.b_{2}(\widetilde{x}+\widetilde{z})+b_{1} \in B\left(\left(x_{0}-z_{0}\right) /(\widetilde{x}-\widetilde{z}),\left(\zeta_{1}+\zeta_{2}\right) /|\widetilde{x}-\widetilde{z}|\right)\right),
$$

where $\left(\zeta_{1}+\zeta_{2}\right) /|\widetilde{x}-\widetilde{z}| \leq\left(\zeta_{1}+\zeta_{2}\right) / \varepsilon$. For all possible values $b_{2}, b_{1} \in \mathbb{Z}$, the lefthand side of (17) defines a lattice in $\mathbb{C}$ with a basis $\{\widetilde{x}+\widetilde{z}, 1\}$ and a determinant

$$
\left|\begin{array}{ll}
\operatorname{Re}(\widetilde{x}+\widetilde{z}) & 1 \\
\operatorname{Im}(\widetilde{x}+\widetilde{z}) & 0
\end{array}\right|=\operatorname{Im}(\widetilde{x}+\widetilde{z}), \quad \text { where } \quad|\operatorname{Im}(\widetilde{x}+\widetilde{z})|>\varepsilon
$$

From well-known estimates for the number of lattice points in a Euclidean circle, we obtain that the number of pairs $\left(b_{2}, b_{1}\right)$ satisfying (77) is bounded from above by a constant of the order $\left(\zeta_{1}+\zeta_{2}\right)^{2} / \varepsilon^{3}$. Let us denote this constant as $\mathbf{c}$. The integer coefficient $b_{0}$ can be uniquely determined from (4) since the right sides of it are less than $1 / 2$. Thus, for a fixed coefficient $b_{4}$ the number of triples $\left(b_{2}, b_{1}, b_{0}\right)$ does not exceed $\mathbf{c}$, and therefore $\sharp \mathcal{R}_{4}\left(b_{4}\right) \leq \mathbf{c} H$.

\section{Proof of Theorem}

Remember that we consider the points

$$
\left(x, z, \omega_{1}, \omega_{2}\right) \in \mathbf{T} \quad \text { and } \quad P \in \mathfrak{P}_{n}\left(H, \mathbf{q}, \mathbf{r}, \mathbf{s}_{1}, \mathbf{s}_{2}\right) .
$$

We prove the theorem for $n \geq 5$. The case $n=4$ follows from Lemma 1 and the Borel-Cantelli lemma.

Definition 2. Let $i_{j} \in\{0,1\}, j=1,2,3,4$. A polynomial $P \in \mathfrak{P}_{n}\left(H, \mathbf{q}, \mathbf{r}, \mathbf{s}_{1}, \mathbf{s}_{2}\right)$ is called $\left(i_{1}, i_{2}, i_{3}, i_{4}\right)$-linear if:

(1) for $\left(i_{1}, i_{2}, i_{3}, i_{4}\right)=(0,0,0,0)$ the system of inequalities

$$
r_{i 1}+s_{i 2} / T<v_{i}+1, \quad i=1,2,3,4,
$$

holds, where $\left(r_{11}, r_{21}, r_{31}, r_{41}\right)=\left(q_{1}, r_{1}, s_{11}, s_{21}\right)$ which are defined in (2), (3)); 


\section{ELLA KOVALEVSKAYA}

(2) for $\left(i_{1}, i_{2}, i_{3}, i_{4}\right)=(1,1,1,1)$ the inequality signs in (8) are reversed;

(3) for $(0,1,1,1)$ the first inequality in (8) has the sign $<$ and the other inequalities have sings $\geq$; and so on. There exist 16 kinds of linear polynomials.

Denote by $\mathfrak{P}_{n}^{\left(i_{1}, i_{2}, i_{3}, i_{4}\right)}$ the class of $\left(i_{1}, i_{2}, i_{3}, i_{4}\right)$-linear polynomials

$$
P \in \mathfrak{P}_{n}\left(H, \mathbf{q}, \mathbf{r}, \mathbf{s}_{1}, \mathbf{s}_{2}\right) .
$$

If $\left(x, z, \omega_{1}, \omega_{2}\right) \in M_{n}(V, \Psi, \Lambda)$ (see $\left.\S 1\right)$, then there exist infinitely many polynomials satisfying at least one of these 16 kinds of linearity. Let $M_{n}^{\left(i_{1}, i_{2}, i_{3}, i_{4}\right)}(V, \Psi, \Lambda)$ denote the set of $\left(x, z, \omega_{1}, \omega_{2}\right) \in \mathbf{T}$ for which the system of inequalities (1) holds for infinitely many polynomials $P \in \mathfrak{P}_{n}^{\left(i_{1}, i_{2}, i_{3}, i_{4}\right)}$. Clearly that

$$
M_{n}(V, \Psi, \Lambda) \quad=\underset{i_{j} \in\{0,1\},(j=1,2,3,4)}{\bigcup} M_{n}^{\left(i_{1}, i_{2}, i_{3}, i_{4}\right)}(V, \Psi, \Lambda) .
$$

Two constants

$$
d_{1}=q_{1}+2 r_{1}+s_{11}+s_{21} \quad \text { and } \quad d_{2}=\left(k_{2}+2 l_{2}+m_{12}+m_{22}\right) / T,
$$

connected with (2), (3), will be used further in our proof.

The proof consists of a series of propositions with different linearity conditions and different ranges of $d_{1}+d_{2}$. They are considered separately. Further, we have

where

$$
\left|P^{\prime}\left(\alpha_{i 1}\right)\right|=H\left|\alpha_{i 1}-\alpha_{i 2}\right| \quad \cdots \quad\left|\alpha_{i 1}-\alpha_{i n}\right|=H^{1-r_{i j}}, \quad i=1,2,
$$

$$
\left(r_{1 j}, r_{2 j}\right)=\left(q_{1}, r_{1}\right) \quad \text { and } \quad\left|P^{\prime}\left(\gamma_{j 1}\right)\right|_{p_{i}}=H^{-s_{j 1}}, \quad j=1,2 .
$$

These relations follow directly from (3).

Proposition 1. Let $P \in \mathfrak{P}_{n}^{(0,0,0,0,)}$. Then $\bar{\mu} M_{n}^{(0,0,0,0)}(V, \Psi, \Lambda)=0$.

P r o o f. According to (8) and (9) we have $d_{1}+d_{2}<n+1$. The proof includes four cases:

(1) $n+\varepsilon \leq d_{1}+d_{2}<n+1$;

(2) $5-\varepsilon \leq d_{1}+d_{2}<n+\varepsilon$;

(3) $\varepsilon \leq d_{1}+d_{2}<5-\varepsilon$;

(4) $d_{1}+d_{2}<\varepsilon$.

We use scheme of the proofs of Proposition 1, 4, 3, 2 of [2], respectively, but there exist some distinctions. The distinctions appear in the sets $\bar{X}=\left(X_{1}, X_{2}, X_{3}, X_{4}\right)$ of the corresponding spaces. Namely, in [2] ones have $\bar{X}=(x, z, \omega) \in \mathbb{R} \times \mathbb{C} \times \mathbb{Q}_{p}$, in our case we have $\bar{X}=\left(x, z, \omega_{1}, \omega_{2}\right) \in \mathbb{R} \times \mathbb{C} \times \mathbb{Q}_{p_{1}}, \times \mathbb{Q}_{p_{2}}$. 


\section{THE CONVERGENCE PART OF A KNINTCHINE-TYPE THEOREM}

Note that in:

(1) we use Lemmas $1-3$ and 5 of $\S 3$;

(2) we use Lemmas 1-5 of $\S 3$ and make a reduction to polynomials of the third degree (in [2] the reduced polynomials have the second degree);

(3) we use Lemmas $1-5$ of $\S 3$ and make a reduction to polynomials $R(t)$ of the fourth degree (in [2] they have the third degree);

(4) we use Lemmas $1-3$ and 5 of $\S 3$ and make a reduction to the polynomials of the third degree (in 2] they have the second degree).

Write some details for (3).

The case of the essential parallelepipeds $\sigma_{4}\left(P_{1}\right), \sigma_{4}\left(P_{2}\right)$ is considered as in 2, p. 205].

For the inessential parallelepipeds $\sigma_{4}\left(P_{1}\right), \sigma_{4}\left(P_{2}\right)$ we have

$R(t)=P_{2}(t)-P_{1}(t)=b_{4} t^{4}+b_{3} t^{3}+b_{2} t^{2}+b_{1} t+b_{0}, \quad b_{i} \in \mathbb{Z}, \quad \operatorname{deg} P=4$,

$R(t)$ is a leading polynomial, i.e., $\left|b_{i}\right| \leq\left|b_{4}\right|, i=0,1,2,3$ (as in [2, p. 205]), and $R(t)$ satisfies the system of inequalities

$$
\begin{gathered}
|R(x)| \ll 2^{-t V_{1}}, \quad|R(z)| \ll 2^{-t V_{2}}, \quad\left|R\left(\omega_{i}\right)\right|_{p_{i}} \ll 2^{-t V_{i+2}}, \quad i=1,2, \\
\left|R^{\prime}(x)\right| \ll 2^{t\left(1-q_{1}+(n-1) \varepsilon_{1}\right)}, \quad\left|R^{\prime}(z)\right| \ll 2^{t\left(1-r_{1}+(n-1) \varepsilon_{1}\right)}
\end{gathered}
$$

at the points $\left(x, z, \omega_{1}, \omega_{2}\right) \in \sigma_{4}(R)=\sigma_{4}\left(P_{1}\right) \bigcap \sigma_{4}\left(P_{2}\right)$, where $X \ll Y$ is equivalent to the notation $X=O(Y)$. Here we have $q_{1} \geq \varepsilon / 3$ and

$$
V_{1}+2 V_{2}+V_{3}+V_{4}=1, \quad V_{i}>0, \quad i=1,2,3,4 .
$$

(see [2, p. 205]). Note that (11) is connected with the system (11) of Theorem (see $\S 1$ ). Also $R(t)$ can be written as

$$
R(t)=b_{4}\left(t-\theta_{1}\right)\left(t-\theta_{2}\right)\left(t-\theta_{3}\right)\left(t-\overline{\theta_{3}}\right)=b_{4} t^{4}+b_{3} t^{3}+b_{2} t^{2}+b_{2} t+b_{0},
$$

where $\theta_{1}, \theta_{2}$ are real roots of $R(t), \theta_{3}, \overline{\theta_{3}}$ are complex roots of it, and

and

$$
\zeta_{3} H(R) \leq\left|b_{4}\right| \leq \zeta_{4} H(R), \quad\left|b_{4}\right|_{p_{j}}>p_{j}^{-n}, \quad j=1,2,
$$

$$
2^{t} \leq H(R)<2^{t+1},
$$

where $\zeta_{j}>0$ is some absolute constant, $j=3,4$. The foundation of this is the same as in [7, Ch. 1, $\S 6$ and Ch. 2, $\S 2$ ] or [2, p. 205].

Note that the number of polynomials $R$ which satisfy (10)-(14) at the points of $\sigma_{4}(R)$ is estimated by Lemma 5 as

$$
<c H(R) .
$$

There are two cases for the polynomial (13): $(\mathbf{a}): \theta_{1} \neq \theta_{2},(\mathbf{b}): \theta_{1}=\theta_{2}$. 


\section{ELLA KOVALEVSKAYA}

Case (a). For the fixed polynomials $P_{1}, P_{2}$ and $R=P_{2}-P_{1}$ we estimate from above the measure $\bar{\mu}(R)$ of the set $\sigma_{4}(R)=\sigma_{4}\left(P_{1}\right) \bigcap \sigma_{4}\left(P_{2}\right)$, where (11) and (12) hold. We have

$$
\left|R^{\prime \prime}\left(\theta_{j}\right)\right| \geq 2\left|b_{4}\left(\theta_{j}-\theta_{3}\right)\left(\theta_{j}-\overline{\theta_{3}}\right)\right| \geq 4 \zeta_{3} \delta^{2} H(R), \quad j=1,2,
$$

where $\delta>0$ is defined in the beginning of $\S 2$. Since $R(t)$ has different roots, we can apply Lemma 1 to it. The third inequality of Lemma 1 , when $j=2$, has the form $\left|x-\theta_{1}\right| \leq 2\left(|R(x)| / \mid R^{\prime \prime}\left(\theta_{1} \mid\right)^{1 / 2}\right.$. Hence the first inequality of (111), (14) and (16)) imply $\left|x-\theta_{1}\right| \ll\left(2^{-t V_{1}} / H(R)\right)^{1 / 2}$. Similarly we have

$$
\left|x-\theta_{2}\right| \ll\left(2^{-t V_{1}} / H(R)\right)^{1 / 2} .
$$

Thus,

$$
\max _{i=1,2}\left(\left|x-\theta_{i}\right|\right) \ll\left(2^{-t V_{1}} / H(R)\right)^{1 / 2} .
$$

Further the first inequality of Lemma 1, the second inequality of (11) and (14) imply

$$
\left|z-\theta_{3}\right| \ll 2^{-t V_{2}} / H(R) .
$$

Let $\rho_{j 1}, \rho_{j 2}, \rho_{j 3}, \rho_{j 4}$ be the $p_{j}$-adic roots of $R(t), j=1,2$. Then the fourth inequality of Lemma 1 , when $j=2$, and the second condition in (13) imply

$$
\left|\omega_{j}-\rho_{j 1}\right|_{p_{j}} \ll\left(2^{-t V_{j+2}}\right)^{1 / 2}, \quad j=1,2 .
$$

Now (17)-(19) and the definition of the measure $\bar{\mu}$ in $\mathcal{O}=\mathbb{R} \times \mathbb{C} \times \mathbb{Q}_{p_{1}} \times \mathbb{Q}_{p_{2}}$ imply $\bar{\mu}(R) \ll 2^{-t A}(H(R))^{-5 / 2}$, where

$$
A=V_{1} / 2+2 V_{2}+V_{3} / 2+V_{4} / 2=\left(V_{1}+2 V_{2}+V_{3}+V_{4}\right) / 2+V_{2} .
$$

We estimate $\sum_{R} \bar{\mu}(R)$, where $R(t)$ is defined in (10)-(14). According to (15), (20) we have

$$
\begin{aligned}
\sum_{R} \bar{\mu}(R) & =\sum_{t=1}^{\infty} \sum_{2^{t} \leq b_{4}<2^{t+1}} \sum_{b_{3}, b_{2}, b_{1}, b_{0}} \bar{\mu}(R) \\
& \ll \sum_{t=1}^{\infty} \sum_{b_{4}=H(R)} H(R) \cdot 2^{-t V_{2}} \cdot 2^{-t\left(V_{1}+2 V_{2}+V_{3}+V_{4}\right) / 2} \cdot(H(R))^{-5 / 2} \\
& \ll \sum_{t} 2^{-t V_{2}} \cdot 2^{-t\left(V_{1}+2 V_{2}+V_{3}+V_{4}\right) / 2} \sum_{2^{t} \leq H(R)<2^{t+1}}(H(R))^{-3 / 2} .
\end{aligned}
$$

According to (12) we get

$$
\sum_{R} \bar{\mu}(R) \ll \sum_{t} 2^{t\left(-V_{2}-1 / 2\right)} \cdot 2^{-t / 2} \ll \sum_{t} 2^{-t\left(1+V_{2}\right)}<\infty .
$$

The Borel-Cantelli Lemma completes the proof of (a). 


\section{THE CONVERGENCE PART OF A KNINTCHINE-TYPE THEOREM}

Case (b). We have

$$
\begin{aligned}
R(t) & =b_{4}\left(t-\theta_{1}\right)^{2}\left(t-\theta_{3}\right)\left(t-\overline{\theta_{3}}\right) \\
& =b_{4} t^{4}+b_{3} t^{3}+b_{2} t^{2}+b_{2} t+b_{0}, \quad b_{i} \in \mathbb{Z} .
\end{aligned}
$$

For the fixed polynomials $P_{1}, P_{2}$ and $R=P_{2}-P_{1}$ with the condition (14) we estimate a measure $\bar{\mu}(R)$ of the set $\sigma_{4}(R)$ where (11), (12) hold. Now (21), (14) imply

$$
\left|x-\theta_{j}\right| \ll\left(2^{-t V_{j}} / \delta^{2}\left|b_{4}\right|\right)^{1 / 2} \ll\left(2^{-t V_{j}} / H(R)\right)^{1 / 2}, \quad j=1,2
$$

$(\delta>0$ is defined in $\S 2)$. Similarly, according to (21), (14) we find

$$
\left|z-\theta_{3}\right| \ll 2^{-t V_{2}} / H(R)
$$

Also we have in $p_{j}$-adic valuation

$$
|R(t)|_{p_{j}}=\left|b_{4}\right|_{p_{j}}\left|t-\rho_{j 1}\right|_{p_{j}}^{2}\left|t-\rho_{j 3}\right|_{p_{j}}\left|t-\rho_{j 4}\right|_{p_{j}} .
$$

Hence (21), (14) imply

$$
\left|\omega_{j}-\rho_{j 1}\right|_{p_{i}} \ll\left(2^{-t V_{j+2}}\right)^{1 / 2}, \quad j=1,2 .
$$

Then

$$
\bar{\mu}(R) \ll 2^{-t A}(H(R))^{-5 / 2},
$$

where $A$ is defined in (20). Further we argue as in (a).

Proposition 2. Let $P \in \mathfrak{P}_{n}^{(1,1,1,1)}$. Then $\bar{\mu} M_{n}^{(1,1,1,1)}(V, \Psi, \Lambda)=0$.

P r o of. According to (8) and (9) we have $d_{1}+d_{2} \geq n+1$. The proof is similar to [2, Proposition 5]. There exists the following distiction: the number of inequalities in all considered systems equals four. The fourth inequality corresponds to the $p_{2}$-adic valuation.

P r o o f of the theorem is based on Propositions 1, 2. The other cases of linearity are the combinations of the two preceding cases with the corresponding coordinates. Namely, the cases $(1,0,0,0)-,(0,1,0,0)-,(0,0,1,0)-,(0,0,0,1)$ -linearity are considered in the same manner since they are the permutations of the coordinates. Thus, it is sufficient to investigate only the $(1,0,0,0)$-linearity case (as well as [2, Proposition 6], where for the second coordinate $i_{2}\left(i_{2}=0\right)$ we add the inequality $\left.q_{21}+k_{22} / T<1+v_{2}+\lambda_{2}\right)$.

The cases $(1,1,0,0)-,(1,0,1,0)-,(1,0,0,1)-,(0,1,1,0)-,(0,0,1,1)-,(0,1,0,1)-$ -linearity are considered in the same manner since they are the permutations of the coordinates. Thus, it is sufficient to investigate only the $(1,0,0,1)$-linearity case (as well as [2, Proposition 7], where for the second coordinate $i_{2}\left(i_{2}=0\right)$ we add the inequality $\left.q_{21}+k_{22} / T<1+v_{2}+\lambda_{2}\right)$.

The cases $(1,1,1,0)-,(1,1,0,1)-,(1,0,1,1)-,(0,1,1,1)$-linearity are considered in the same manner since they are also the permutations of the coordinates. 


\section{ELLA KOVALEVSKAYA}

Thus, it is sufficient to investigate only the $(1,1,1,0)$-linearity case. It is a combination of [2, Proposition 6,7], where for the second coordinate $i_{2}\left(i_{2}=1\right)$ we add the inequality $q_{21}+k_{22} / T \geq 1+v_{2}+\lambda_{2}$, and for the third coordinate $i_{3}$ $\left(i_{3}=1\right)$ we take $r_{1}+l_{2} / T \geq 1+v_{3}+\lambda_{3}$.

Theorem is proved. Note that the similar method was used earlier in [5].

Acknowledgements. The author is grateful to Professor N. K a los ha and Professor D. Vasilyev for the discussion, and to the referee for the useful remarks. Note that the mentioned proof of Lemma 5 was proposed by Professor N. Kalosha. It is shorter than the author's one.

\section{REFERENCES}

[1] BERNIK, V. I.: Simultaneous approximation of zero by integer polynomials, Izv. Academy Sci. SSSR. Ser. Math. 44 (1980), 24-45.

[2] BUDARINA, N.-DICKINSON, D.- BERNIK, V.: Simultaneous Diophantine approximation in the real, complex and $p$-adic fields, Math. Proc. Cambridge Phil. Soc. 149 (2010), 193-216.

[3] BERNIK, V. I.-KALOSHA, N. I.: Approximation of zero by integer polynomials in space $\mathbb{R} \times \mathbb{C} \times \mathbb{Q}_{p}$, Proc. National Academy Sci. Belarus. Phis. and Math. Ser. 1 (2004), 121-123.

[4] BERESNEVICH, V. V.-BERNIK, V. I.-KOVALEVSKAYA, E. I.: On approximation of p-adic numbers by p-adic algebraic numbers, J. Number Theory 111 (2005), 3-56.

[5] KOVAlEVSKAYA, E.: Diophantine approximation in $\mathbb{C} \times \mathbb{Q}_{p}$, : in: Analytic and Probab. Methods in Number Theory (A. Laurinčikas et al., eds.), Proc. of the 4th Intern. Conf. in Honour of J. Kubilius, Palanga, Lithuania, 2006, TEV, Vilnius, 2007, pp. 56-71.

[6] KOVALEVSKAYA, E.: Simultaneous Diophantine approximation in $\mathbb{R}^{2} \times \mathbb{C} \times \mathbb{Q}_{p}$, Tatra Mt. Math. Publ. 56 (2013), 79-86.

[7] SPRINDŽUK, V. G.: Mahler's Problem in the Metric Theory of Numbers. Sci. and Technics, Minsk, 1965 (In Russian); AMS Transl. of Math. Monographs, Vol. 25, Amer. Math. Soc., Providence, R. I., 1969.

[8] Z̆ELUDEVICH, F.: Simultane diophantishe Approximationen abhängiger Grössen in mehreren Metriken, Acta Arithm. 46 (1986), 285-296.

[9] BERNIK, V. I.-DODSON, M. M.: Metric Diophantine Approximation of Manifolds, in: Cambridge Tracts in Math., Vol. 137, Cambridge University Press, Cambridge, 1999.

[10] MOHAMMADI, A.-SALEHI GOLSEFIDI, A.: Simultaneous diophantine approximation in non-degenerate p-adic manifolds. Israil J. Math.188 (2012), 231-258.

Received August 7, 2014

Byelorussian State Agricultural
Technic University
Nezavisimosty 99
BY-220023-Minsk
BELARUS
E-mail: ekovalevsk@mail.ru kovalevsk@im.bas-net.by 